\title{
Fluid-structure interactions of fast photomechanical liquid crystal elastomers driven by light
}

\author{
J. Bin ${ }^{1}$, W. S. Oates ${ }^{2}$ \& M. Y. Hussaini ${ }^{1}$ \\ ${ }^{1}$ Department of Mathematics, Florida State University, USA \\ ${ }^{2}$ Department of Mechanical Engineering, Florida State University, USA
}

\begin{abstract}
A new class of photomechanical liquid crystal elastomers (LCE) has emerged, which generate large bending deformation and fast response times that scale with the resonance of the elastomer films. These films are classified as glassy elastomers (modulus $\sim 1 \mathrm{GPa}$ ) and are doped with photoresponsive azobenzene liquid crystals to provide novel light induced deformation. These materials are promising for developing propulsions systems for insect size aircraft and microfluidic devices, for example. The photomechanical efficiency of these materials in a fluid medium is of high interest to understand the performance attributes of this class of smart materials. Here, a numerical study is presented that describes the photomechanical structural dynamic behaviour in a fluid medium. We simulate the oscillation of photomechanical cantilevers excited by light while simultaneously modeling the effect of the surrounding fluid at different ambient pressures. The photoelastomer structure is modeled as a thin plate and coupled with photomechanical constitutive relations to compute the transverse displacement. For the fluid, three dimensional unsteady incompressible Navier-Stokes equations using the arbitrary Lagrangian Eulerian (ALE) form are used to consider dynamic mesh movement on a local mesh and boundary conditions on the elastomer material interface. The fluid equations are discretized using a conventional finite volume method (FVM) on a structured curvilinear coordinate system. Numerical examples are given which provide new insight into photomechanical material efficiencies in a fluid medium as a function of ambient pressure.

Keywords: liquid crystal elastomer, azobenzene LCN, arbitrary LagrangianEulerian, artificial compressibility, fluid-structure interaction.
\end{abstract}




\section{Introduction}

Liquid crystal elastomers (LCEs) are active materials that combine the characteristics of elastomers with liquid crystals [1]. These characteristics result in an interesting coupling between the liquid crystal active medium and deformation of the polymer network that arises from a variety of external stimuli. The change of the internal liquid crystal order can be driven by external stimuli such as heat, light, $\mathrm{pH}$, polarity, humidity, and mechanical loads which provides interesting opportunities for developing smart material actuators and sensors.

Of particular interest is azobenzene LCNs (azo-LCNs) which can convert light energy into mechanical work on a polymer network, which were first considered by Lovrien [2] in 1967. Laser controlled active polymers provide a unique adaptive material for micro air vehicles, microfluidic systems, and adaptive robotic skins. These materials consist of azobenzene liquid crystals that act as molecular motors and do work on the host polymer network. During light stimuli, a complex photoisomerization process (light induced liquid crystal structure transformations) induces bending of the photoelastomer film due to light absorption and strain gradients through the film thickness. Figure 1 illustrates an azobenzene LCE film where the rod-like molecules undergo a trans to cis isomerization when they absorb photons. The cis conformation results in the shape change of the molecule from rod-like to bent, which changes the nematic ordering. Two liquid crystal transformations are believed to occur: 1) trans-cis photoisomerization (rod to kinked molecular structure change) from UV light which produces order-disorder behaviour in monodomain specimens and 2) trans-cis-trans (order-disorder-reorder) process which includes a rotation of the rod shaped liquid crystals due to polarized light with a wavelength of approximately $442 \mathrm{~nm}$. The latter photoisomerization process is motivated by surface relief grating experiments and recent free standing experiments $[3,4]$.

These materials are promising for developing propulsions systems for insect size aircraft and microfluidic devices. Therefore, photomechanical efficiency of these materials in a fluid medium is of high interests to understand performance attributes of this class of smart materials. The objective of this study is to develop a computational method for the fluid-structure interaction (FSI) analysis of fast photomechanical liquid crystal elastomers driven by light. Here, a numerical study is presented that describes the photomechanical structural dynamic behaviour in a fluid medium.
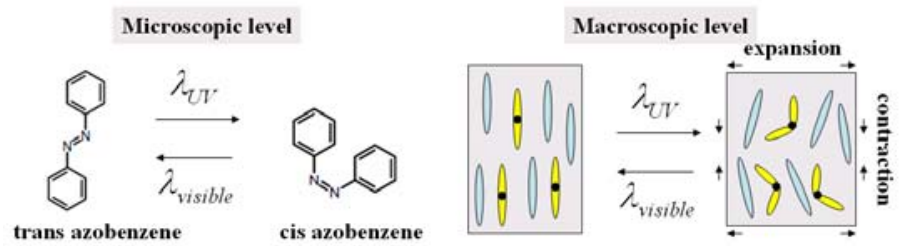

Figure 1: The schematic of azobenzene LCE showing a photostrain. 
We simulate the oscillation of photomechanical cantilevers excited by light by simultaneously modeling the effect of the surrounding fluid at different ambient pressures. To this end, an efficient and stable FSI model is developed to address the interactions between the incompressible fluid and the photomechanical elastomer during light stimuli. Light absorption is modelled by assuming isotropic absorption characteristics, which results in an exponential attenuation of the optical intensity through the thickness of the film known as the Beer-Lampert law [5].

The application of numerical methods to the solution of a coupled field problem results in a system of nonlinear algebraic equations, which may be resolved according to a partitioned approach in this study [7-9]. These methods are found to be very practical because they allow the modularity of well developed computational techniques to be retained on each physical domain. This work adopts a partitioned, boundary-fitted arbitrary Lagrangian-Eulerian (ALE) approach to couple the fluid and the photomechanical solid. The solid structure is resolved in a Lagrangian description by using cubic B-spline shape functions and a 2nd order implicit Newmark central scheme for time integration in order to accurately model the photomechanical bending. For the fluid domain, three dimensional unsteady incompressible Navier-Stokes equations using the arbitrary Lagrangian Eulerian (ALE) form are numerically implemented along with kinematic boundary conditions to ensure compatibility at the interface. The approach also includes geometric conservation on the deforming fluid domain to improve numerical accuracy. The fluid equations are discretized using a conventional finite volume method (FVM) on structured curvilinear coordinate system. For the movement of the computational mesh, an adaptive structured mesh redistribution method (ASMRM) is introduced here and proper monitor functions are suggested to obtain an optimal grid distribution on a complicated geometry with non-uniformly distributed grid points [10]. Numerical examples are given which provide new insight on photomechanical material efficiencies in a fluid medium as a function of ambient pressure. The assessment of ambient pressure on photomechanical efficiency is motivated by recent experimental results that show significant differences in light induced flapping behaviour [4].

\section{Computation of a photostrain in azo-LCN fueled by light}

The photoactuated LCE is modelled as a photomechanically coupled, isotropic thin film. Light attenuation through the film thickness results in an exponential attenuation of the optical intensity and subsequently, photomechanical bending [5]. By assuming isotropic absorption, light attenuation is governed by

$$
I(z)=I_{0} e^{-(z+h / 2) / d}
$$

where $I_{0}$ is the light at the top surface and $d$ is absorption coefficient. As photons are absorbed, the rod-like molecule structure undergoes an isomerization. This transition results in a contraction or expansion of the photoelastomer material. In the constitutive model that couples light and strain, 
the photostrain is assumed to be proportional to the optical intensity. This photostrain is also assumed to be volume-conserving with in-plane components given by

$$
\varepsilon_{i j}^{p h}(z)=\frac{\varepsilon^{p h}(z)|\cos \theta|}{4}\left[\begin{array}{lll}
1+3 \cos (2 \theta) & 1-3 \cos (2 \theta) & 6 \sin \theta \cos \theta
\end{array}\right]^{T}
$$

where $\theta$ is the angle between the light polarization and the nematic axis of the trans azo-benzene liquid crystals. Following Eq. (1), the thickness dependence is defined by $\varepsilon^{p h}(z)=-\varepsilon_{p s} \exp [-(z+h / 2) / d]$ where $\varepsilon_{p s}$ is the strain at the top surface $(z=-h / 2)$. In all subsequent analysis, $\theta=0^{\circ}$, and Eq. (2) simplifies to

$$
\varepsilon_{i j}^{p h}(z)=\left\{\begin{array}{lll}
\varepsilon_{x}^{p h} & \varepsilon_{y}^{p h} & \gamma_{x y}^{p h}
\end{array}\right\}^{T}=\left\{\begin{array}{lll}
\varepsilon^{p h}(z) & -\varepsilon^{p h}(z) / 2 & 0
\end{array}\right\}^{T} .
$$

Dunn developed an equivalent bilayer model to facilitate finite element implementation [6]. The photostrain driven by light is only defined in layer 1 according to

$$
\varepsilon^{p h}(z)=\left\{\begin{array}{cc}
-\varepsilon_{p l} & \text { in layer } 1 \\
0 & \text { in layer } 2
\end{array}\right.
$$

Figure 2 shows the schematic of exponential attenuation of the optical intensity through the plate thickness and the configuration of bilayer model. Through the force and moment valance relations, two unknowns are analytically calculated, which are given by [6]

$$
\varepsilon_{p l}=\frac{d\left(e^{h / d}-1\right)^{2} \varepsilon_{p s}}{2\left[d\left(e^{h / d}-1\right)-h\right] e^{h / d}}, \quad h_{1}=\frac{2\left[d\left(e^{h / d}-1\right)-h\right]}{e^{h / d}-1}
$$

These constitutive relations are coupled with elasticity and structural dynamic bending equations in the following section.

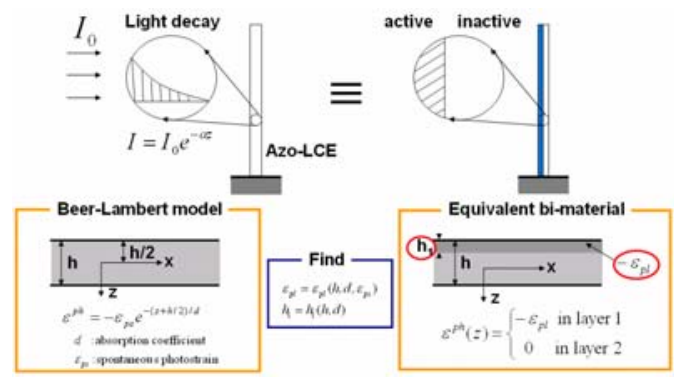

Figure 2: Exponential attenuation of the optical intensity through the thickness of the plate and bilayer model. 


\section{Structural dynamic-photoelastomer plate solver}

\subsection{Photoelastomer plate governing equations}

The governing equations for a thin rectangular photomechanical plate can be determined by balancing forces and moments and coupling the balance relations to elasticity and photomechanical coupling described in the preceding section. In this study, small displacements are assumed which results in decoupled transverse and in-plane displacements. The resulting transverse displacement plate equation is expressed for a plate of length $l$, width $d$, and thickness $h$ as

$$
\rho_{s} \frac{\partial^{2} w}{\partial t^{2}}-\frac{\partial^{2} M_{x}}{\partial x^{2}}-\frac{\partial^{2} M_{y}}{\partial y^{2}}-2 \frac{\partial^{2} M_{x y}}{\partial x \partial y}=f_{n}, \quad \Omega=[0, l] \times[0, d]
$$

where $w$ is the displacement along the z-direction, $f_{n}$ is the external pressure, $\rho_{s}$ is the density and the moment components are $M_{x}, M_{y}$, and $M_{x y}$ which include elastic, damping, and photomechanical induced moments. The moment equations are defined by

$$
\left[\begin{array}{c}
M_{x} \\
M_{y} \\
M_{x y}
\end{array}\right]=\int_{-\frac{h}{2}}^{\frac{h}{2}}\left[\begin{array}{c}
\sigma_{x x} \\
\sigma_{y y} \\
\sigma_{x y}
\end{array}\right] z d z
$$

where the constitutive relations are given by

$$
\sigma_{\mathrm{x}}=\frac{E}{\left(1-v^{2}\right)}\left(\left(\varepsilon_{x x}-\varepsilon_{x x}^{p h}\right)+v\left(\varepsilon_{y y}-\varepsilon_{y y}^{p h}\right)\right)+\frac{c}{\left(1-v^{2}\right)}\left(\dot{\varepsilon}_{x x}+v \dot{\varepsilon}_{y y}\right)
$$

where $E$ is Young's modulus, $v$ is the Poisson's ratio, and $c$ is the KelvinVoigt damping coefficient. The strain rates are denoted by $\dot{\varepsilon}_{x x}$ and $\dot{\varepsilon}_{y y}$ for this stress component. Similar relations exist for the stress components $\sigma_{y y}$ and $\sigma_{x y}$; see Smith [11] for details.

The plate is assumed to have a fully clamped edge condition at $x=0$ and free boundary conditions along all other sides. Physical values used for the elastic characteristics of the photoelastomer film are $\rho_{s}=1000 \mathrm{~kg} / \mathrm{m}^{3}, v=0.33$, $E=2 \times 10^{9} \mathrm{~N} / \mathrm{m}^{2}$, and $c=2.5 \times 10^{-4} \mathrm{E}$.

\subsection{Numerical methods}

In this study, we use cubic B-splines to the construct approximating subspace. Equation (6) can be expressed as the following the weak model formulation.

$$
\int_{\Omega}\left[\rho_{s} \frac{\partial^{2} w}{\partial t^{2}} \varphi-M_{x} \frac{\partial^{2} \varphi}{\partial x^{2}}-M_{y} \frac{\partial^{2} \varphi}{\partial y^{2}}-2 M_{x y} \frac{\partial^{2} \varphi}{\partial x \partial y}-f_{n} \varphi\right] d \omega=0
$$

where $\varphi$ in the space of test functions is given by 


$$
\begin{array}{r}
V=H_{0}^{2}(\Omega)=\left\{\varphi \in H^{2}(\Omega) \mid \varphi(0, y)=\varphi_{x}(0, y)=0 \text { for } 0 \leq y \leq d\right. \\
\left.\varphi(x, 0)=\varphi_{y}(x, 0)=0 \text { for } 0 \leq x \leq l\right\}
\end{array}
$$

To formulate approximate solutions based on cubic B-splines, the domain is partitioned equally, $\left(N_{x}, N_{y}\right)=(8,8)$. To accommodate the essential boundary conditions $w(t, 0)=\frac{\partial w}{\partial x}(t, 0)=0$, we employ the basis functions

$$
\varphi_{j}(x)= \begin{cases}\hat{\varphi}_{0}(x)-2 \hat{\varphi}_{-1}(x)-2 \hat{\varphi}_{1}(x), \mathrm{j}=1 \\ \hat{\varphi}_{j}(x) & , \mathrm{j}=2, \cdots, \mathrm{N}+1\end{cases}
$$

which are modified to ensure that $\varphi_{j} \in V$ for $j=1, \cdots, N+1$ where $\hat{\varphi}_{j}(x)$ is a standard cubic B-spline. Using the definition (11), we define modified cubic spline basis functions $\varphi_{m}(x)$ and $\varphi_{n}(y)$ on the intervals $[0, l]$ and $[0, d]$.

Approximate displacements have the representation given by

$$
w^{N}(t, x, y)=\sum_{k=1}^{N_{w}} w_{k}(t) \Phi_{k}(x, y)=\sum_{m=1}^{N_{x}+1} \sum_{n=1}^{N_{y}+3} w_{m n}(t) \varphi_{m}(x) \varphi_{n}(y) .
$$

Substitution of eqn (12) into eqn (9) yields the vector-valued system

$$
\mathbf{M} \ddot{\mathbf{w}}+\mathbf{Q} \dot{\mathbf{w}}+\mathbf{K w}=\mathbf{f}
$$

where $\mathbf{w}(t)=\left[w_{1}(t), \cdots, w_{N_{w}}(t)\right]^{T}$. The mass and stiffness matrices are given by the reference [11]. The damping matrix $\mathbf{Q}$ is constructed as $\mathbf{Q}=2.5 \times 10^{-4} \mathbf{K}$ to match the damping characteristic of the LCE film.

The equation is advanced by means of a Newmark time integration scheme in conjunction with a Newton-Raphson iterative solution procedure [9].

\section{Unsteady incompressible fluid flow solver}

\subsection{Equations of a fluid flow}

The fluid governing equations are the nonlinear time-dependent incompressible Navier-Stokes equations of a laminar, constant viscosity flow without body forces. Introducing the artificial compressibility to connect pressure with a continuity equation [12], the equations of motion of the fluid can be compactly written in generalized curvilinear coordinate system as

$$
\begin{aligned}
& \frac{\partial \widetilde{\mathbf{Q}}}{\partial \tau}+\mathbf{I}_{p} \frac{\partial \widetilde{\mathbf{Q}}}{\partial t}+\frac{\partial}{\partial \xi^{k}}\left(\widetilde{\mathbf{E}}^{k}-\widetilde{\mathbf{E}}_{v}^{k}\right)=0, \\
& \tilde{\mathbf{Q}}=\mathbf{Q} / J=\left[\begin{array}{llll}
p & u_{1} & u_{2} & u_{3}
\end{array}\right]^{T} / J \text {, } \\
& \widetilde{\mathbf{E}}^{k}=\frac{1}{J}\left[\beta\left(U^{k}-\xi_{t}^{k}\right) \quad u_{1} U^{k}+p \xi_{x_{1}}^{k} \quad u_{2} U^{k}+p \xi_{x_{2}}^{k} \quad u_{3} U^{k}+p \xi_{x_{3}}^{k}\right]^{T},
\end{aligned}
$$

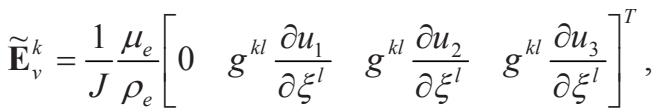


where $\mathbf{I}_{p}=\operatorname{diag}[0,1,1,1],\left[x_{1}, x_{2}, x_{3}\right] \equiv[x, y, z]$ and $\left[\xi^{1}, \xi^{2}, \xi^{3}\right] \equiv[\xi, \eta, \zeta]$ are the Cartesian coordinates and the computational coordinates, respectively, $t$ and $\tau$ are the physical and the pseudo time, respectively, $p$ is the static pressure divided by the density, $u_{k}$ are the Cartesian velocity components, $U^{k}\left(=\xi_{t}^{k}+u_{m} \xi_{x_{m}}^{k}\right)$ are the contravariant velocity components and $\xi_{x_{m}}^{k}$ are the metrics of the geometric transformation, $\xi_{t}^{k}$ are the mesh velocities, $J$ is the Jacobian of the geometric transformation, $g^{k l}$ is the contravariant metric tensor given as $g^{k l}=\xi_{x_{m}}^{k} \xi_{x_{m}}^{l}, \widetilde{\mathbf{E}}^{k}$ are the inviscid fluxes, $\widetilde{\mathbf{E}}_{v}^{k}$ are the viscous fluxes.

Due to the fact that different ambient pressures are studied to quantify its influence on photomechanical bending dynamics, a correlation between the Knudsen number and viscosity is introduced. The viscosity is directly dependent upon the pressure and does change with the Knudsen number, since a high $K n$ means that the gas experiences very few collisions and that the flow is not viscous anymore. In this study, a well-known dynamic viscosity model derived by Veijola et al. [13] is used, $\mu_{e}=\mu_{f} /\left[1+9.638 \mathrm{Kn}^{1.159}\right]$ where $\mu_{f}=1.82 \times 10^{-5} \mathrm{Ns} / \mathrm{m}^{2}$ is the dynamic viscosity at ambient condition. The Knudsen number can be simply given by $K n=\lambda / \Delta=\left(\lambda_{a m b} / \Delta\right)\left(p_{a m b} / p_{e}\right)$ where $\Delta$ is the characteristic length (i.e., length of the cantilever) and the subscripts $e$ and $a m b$ denote the condition of interest and the ambient condition, respectively. The density is determined by the ideal gas law given by $\rho_{e}=p_{e} / R T_{a m b}$.

\subsection{Numerical methods for the fluid flow}

To implement the time evolution of the resulting hyperbolic-parabolic system (14), the physical time derivative is discretized using a second-order backward difference method and the pseudo-time derivative is discretized by an implicit backward finite difference method. The diffusion terms are evaluated by the standard second-order central finite difference scheme whereas the inviscid terms are computed by the flux vector splitting method in conjunction with van Leer's MUSCL formulation for high order spatial accuracy [14]. The implicit approximately factored scheme of Beam and Warming (AF-ADI) is incorporated to solve a system of block tri-diagonal matrix $[15,16]$.

\section{Grid movement technique}

\subsection{Adaptive structured mesh redistribution method}

An adaptive structured mesh redistribution method (ASMRM) is used to make a moving mesh for a fluid part [10], which is given by

$$
\frac{\partial x_{i}}{\partial \tau^{\prime}}+\frac{1}{J} \frac{\partial x_{i}}{\partial \xi^{m}} \frac{\partial}{\partial \xi^{n}}\left(\frac{J \mathbf{g}^{m n}}{\omega_{m}}\right)=0, m, n=1,2,3
$$


where $\tau^{\prime}$ represents the pseudo time and $\mathbf{g}^{i j}$ and $\mathbf{g}_{i j}$ denote contravariant and covariant metric tensors, respectively [10]. The monitor functions, $\omega_{m}$, are used to control the spacing and orientation of the grid. Based on the initial mesh configuration and boundary point distribution, the monitor functions are determined by

$$
\omega_{1}=\rho_{\xi}^{\prime} h_{\eta}^{2} h_{\zeta}^{2} / J, \omega_{2}=\rho_{\eta}^{\prime} h_{\xi}^{2} h_{\zeta}^{2} / J, \omega_{3}=\rho_{\zeta}^{\prime} h_{\xi}^{2} h_{\eta}^{2} / J
$$

where

$$
\begin{gathered}
h_{\xi}=\left(x_{\xi}^{2}+y_{\xi}^{2}+z_{\xi}^{2}\right)^{1 / 2}, h_{\eta}=\left(x_{\eta}^{2}+y_{\eta}^{2}+z_{\eta}^{2}\right)^{1 / 2}, h_{\zeta}=\left(x_{\zeta}^{2}+y_{\zeta}^{2}+z_{\zeta}^{2}\right)^{1 / 2}, \\
\rho_{\xi}^{\prime}=1 /\left[\sum_{i=1}^{L}\left(h_{\xi}\right)_{i, j, k} / L\right], \rho_{\eta}^{\prime}=1 /\left[\sum_{j=1}^{M}\left(h_{\eta}\right)_{i, j, k} / M\right], \rho_{\zeta}^{\prime}=1 /\left[\sum_{k=1}^{N}\left(h_{\zeta}\right)_{i, j, k} / N\right]
\end{gathered}
$$

and $(L \times M \times N)$ denote the mesh sizes in the $\xi$-, $\eta$ - and $\zeta$-directions, respectively.

\section{Numerical fluid-structure coupling scheme}

\subsection{Block-Gauss-Seidel method}

Following Farhat and Lesoinne [8], a staggered solution procedure with full convergence subiteration at each time step is used to ensure strong coupling of the partitioned fluid and structure fields. As shown in Fig. 3, from the mesh information ( $\mathbf{u}_{n}$ and $\dot{\mathbf{u}}_{n}$ ), new mesh information at time level $(n+1 / 2)$ is obtained. This information is used for the flow computation at time $(n+1 / 2)$ in an implicit manner. The pressure field on the interface is extracted and transferred into a structural code as an external pressure. The structural system advances using the second-order time accurate scheme in an implicit manner. When implemented in conjunction with the implicit dual time stepping scheme of the fluid solver and the implicit Newmark/Newton-Raphson scheme of the structural solver this subiterative approach is particularly attractive as it enables a large physical time step to be applied without impairing the long term stability of the coupled solution $[8,9]$.

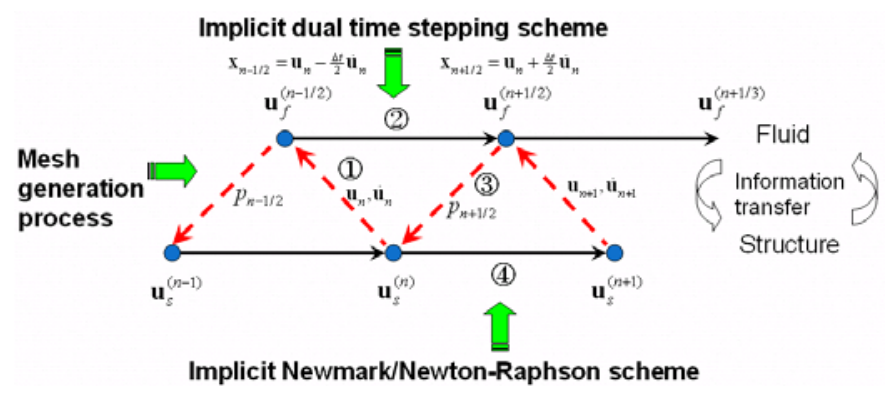

Figure 3: Numerical fluid-structure coupling procedure. 


\subsection{Geometric conservation law}

In time-dependent deforming meshes, a discrete Geometric Conservation Law (dGCL) that is similar in its principle to the GCL condition first mentioned by Thomas and Lombard [17] must be satisfied to eliminate metric cancellation errors and to ensure freestream preservation. This can be derived from the scalar mass conservation equation.

$$
\frac{\partial\left(J^{-1}\right)}{\partial t}+\frac{\partial}{\partial \xi^{k}}\left(\xi_{t}^{k}\right)=0
$$

where $\xi_{t}^{k} / J=-\left[\dot{x}\left(\xi_{x}^{k} / J\right)+\dot{y}\left(\xi_{y}^{k} / J\right)+\dot{z}\left(\xi_{z}^{k} / J\right)\right]$ and $(\dot{x}, \dot{y}, \dot{z})$ is mesh velocities in the fluid domain. In order to satisfy the GCL with a second-order time accurate scheme, the following discretizations are used [8].

$$
\mathbf{x}_{n-1 / 2}=\mathbf{u}_{n}-\frac{\Delta t}{2} \dot{\mathbf{u}}_{n}, \quad \mathbf{x}_{n+1 / 2}=\mathbf{u}_{n}+\frac{\Delta t}{2} \dot{\mathbf{u}}_{n}
$$

with given initial conditions $\mathbf{u}_{0}$ and $\dot{\mathbf{u}}_{0}$.

From Eq. (23), $\mathbf{u}_{n}=\mathbf{x}_{n}$ and $\dot{\mathbf{u}}_{n}=\dot{\mathbf{x}}_{n}$ are automatically satisfied on the interface boundary between the solid and the fluid. Finally, the Jacobian is updated by the following equation.

$$
(1 / J)^{n+1 / 2}=(1 / J)^{n-1 / 2}+\Delta t R^{n}\left(\dot{x}, \dot{y}, \dot{z}, \xi_{x}^{k}, \xi_{y}^{k}, \xi_{z}^{k}, J\right)
$$

where $R=-\partial\left(\xi_{t}^{k}\right) / \partial \xi^{k}$. The residual $R$ is discretized with the same method as the flow equations of Eq. (14).

\section{Results and discussions}

\subsection{Flow induced vibration of a elastic cantilever}

The first example is chosen to demonstrate the ability of the numerical model to treat complex flow-structure interaction problems representing small to moderate bending deformations with no light stimulus present. Geometry, boundary conditions, and the material parameters of the fluid and structure are given in Ref. [9]. The mesh size used is $\left(N_{x}, N_{y}, N_{z}\right)=(141,3,101)$. The time step used here is $\Delta t=1.12 \times 10^{-3} .30$ sub-iterations are performed at every physical time step to obtain converged values. Figure 4 shows instantaneous mesh configuration of the FSI and the displacement at the tip of the cantilever in time. Figure 5 represents instantaneous pressure and streamwise velocity contours. As shown in Table 1, very good agreement with results in references $[9,18]$ is obtained from the simulation. 

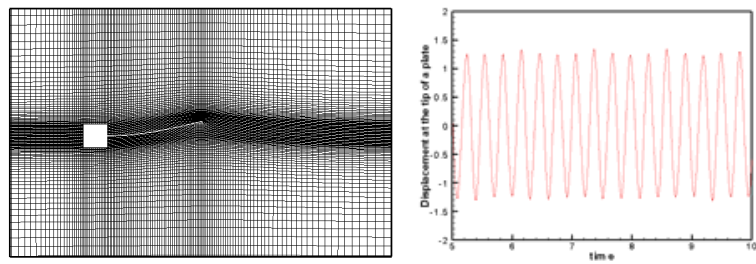

Figure 4: Instantaneous mesh configuration and displacement at the tip in time.
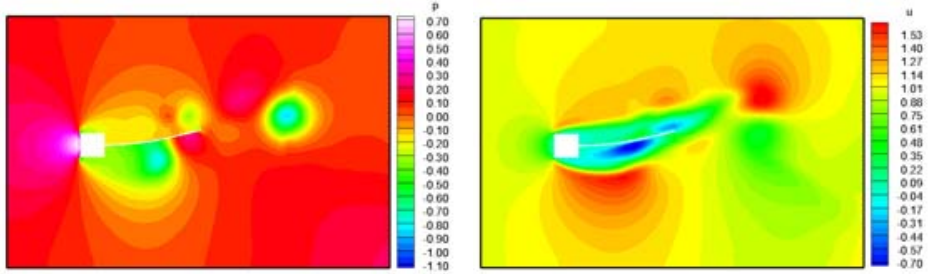

Figure 5: Instantaneous pressure and streamwise velocity contours.

Table 1: $\quad$ Comparison of current results with those in the literature.

\begin{tabular}{|l|c|c|}
\hline Author & Period of oscillation T (s) & Tip deflection $d_{\max }(\mathrm{m})$ \\
\hline Wall [18] & $0.31-0.36$ & $1.12-1.32$ \\
\hline Wood et al. $[9]$ & $0.32-0.36$ & $1.10-1.20$ \\
\hline Current work & 0.32 & $1.24-1.34$ \\
\hline
\end{tabular}

\subsection{Effect of the surrounding fluid at different ambient pressure}

Figure 6 represents the physical model and boundary conditions used for the photomechanical fluid-structure computation. To simplify the problem, we perform two dimensional fluid-structure interaction computations. All mesh configurations are the same as the validation problem described above. All the lengths are re-sized to match the length of the LCE film of interest. Since there is no flow in the computation domain, all the physical values in the regions where radiation boundary condition should be applied are simply extrapolated from the interior ones. In contrast to the experiments given in [4], a periodic light source by a laser having the same frequency as the resonance frequency of the film is imposed on the cantilever surface of the film, which will results in a sinusoidal photostrain. This simplifies the light-matter boundary conditions due to complexities associated with the film bending over $180^{\circ}$ through the light source. This creates non-Snell like reflection and absorption on the film surface and photostrictive bending on both sides of the film during large rotation. The external sinusoidal light stimulus approximates this behaviour. The time step 


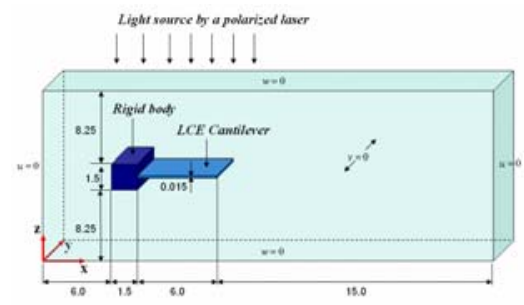

Figure 6: $\quad$ Physical model and boundary conditions.

used here is $\Delta t=1.25 \times 10^{-4}$. When a time-periodic light source is applied, the cantilever starts to oscillate, but its motion would be prohibited by the surrounding air. Figure 7 shows oscillation at the tip by the external sinusoidal light source in time with respect to pressure variation on three different geometric configurations. It is observed that as the geometry increases, the damping effect by the surrounding fluid is increased and a phase shift also occurred.

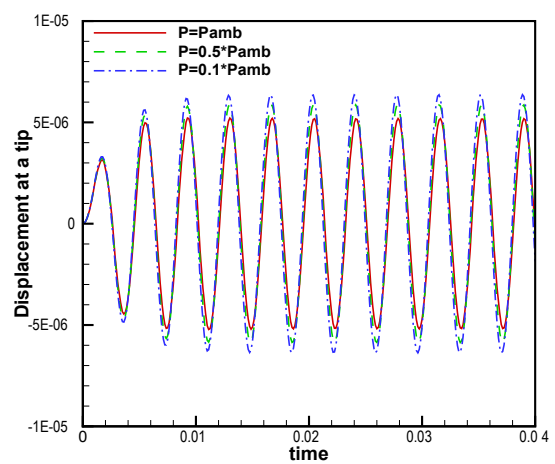

(a) $l \times b=1.5 \mathrm{~mm} \times 0.25 \mathrm{~mm}$,

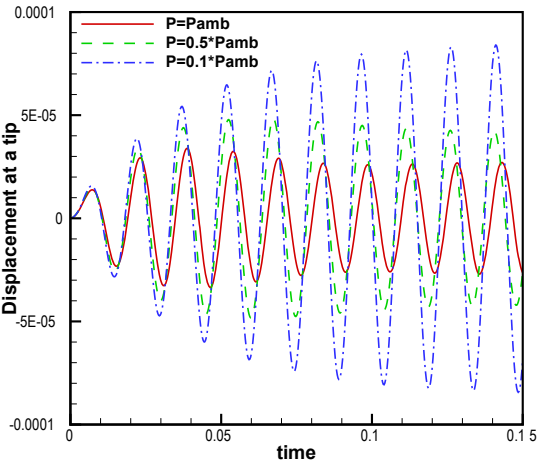

(b) $l \times b=3 m m \times 0.5 m m$,

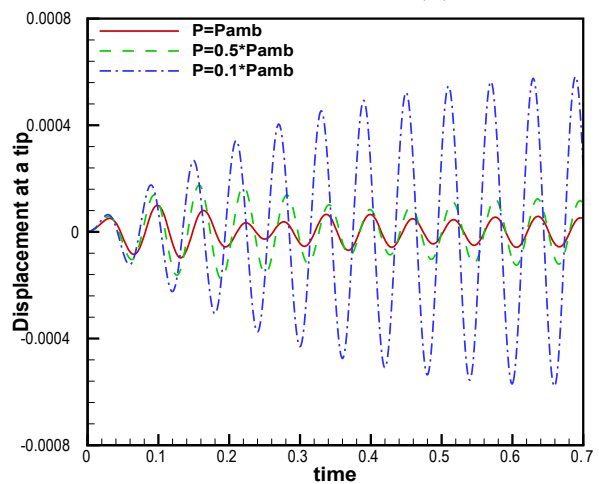

(c) $l \times b=6 m m \times 1 m m$.

Figure 7: Oscillations at the tip by the external sinusoidal light with respect to the pressure variation. 


\section{Summary and future work}

An efficient and stable FSI model was developed to deal with the interaction between the incompressible fluid and the flexible body due to the photo-light interactions. We simulated the oscillation of photomechanical cantilevers excited by light and examined the effect of the surrounding fluid at different ambient pressures and sizes of the film on its motion. It is shown that the size of the photoelastomer is sensitive to the amount of light induced deformation under different ambient pressures suggesting different amounts of photomechanical work are transferred to the fluid.

Ongoing work will extend these results to a fully coupled 3D LCE body showing large flexible flapping motion by light to unravel the mechanical and aerodynamic physics underlying their interaction.

\section{Acknowledgement}

The authors gratefully acknowledge support through the DARPA YFA grant N66001-09-1-2105. Any opinions, findings, and conclusions or recommendations expressed in this publication are those of the authors and do not necessarily reflect the views of the funding sponsors.

\section{References}

[1] Warner, M. \& Terentjev, E., Liquid Crystal Elastomers, Oxford University Press, 2007.

[2] Lovrien, R., The photoviscosity effect, Proceedings of the National Academy of Sciences of the United States of America, 57(2), pp. 236-242, 1967.

[3] White, T. J., Serak, S. V., Tabiryan, N. V., Vaia, R. A. \& Bunning, T. J., Polarization controlled, photodriven bending in monodomain liquid crystal elastomer cantilevers, Journal of Materials Chemistry, 19, pp. 1080-1085, 2009.

[4] S. Serak, S., Tabiryan, N., Vergara, R., White, T. J., Vaia, R. A. \& Bunning, T. J., Liquid crystalline polymer cantilever oscillators fueled by light, Soft Matter, 6, pp. 779-783, 2010.

[5] Corbett, D. \& Warner, M., Bleaching and stimulated recovery of dyes and of photocantilevers, Physical Review E, 77, pp. 051710, 2008.

[6] Dunn, M. L., Photomechanics of mono- and polydomain liquid crystal elasotmer films, Journal of Applied Physics, 102, pp. 013506-013506-7, 2007.

[7] Gordnier, R. E., High fidelity computational simulation of a membrane wing airfoil, Journal of Fluids and Structures, 25, pp. 897-917, 2009.

[8] Farhat, C. \& Lesoinne, M., Two efficient staggered algorithms for the serial and parallel solution of three-dimensional nonlinear transient aeroelastic problems, Computer Methods in Applied Mechanics and Engineering, 182, pp. $499-515,2000$. 
[9] Wood, C, Gil, A. J., Hassan, O. \& Bonet, J., Partitioned block-Gauss-Seidel coupling for dynamic fluid-structure interaction, Computers and Structures, 88, pp. 1367-1382, 2010.

[10] Bin, J., Uzun, A. \& Hussaini, M. Y., Adaptive mesh redistribution method for domains with complex boundaries, Journal of Computational Physics, Revised, 2010.

[11] Smith, R. C., Smart material systems: Model development, Frontiers in Applied Mathematics Series 32, SIAM, 2005.

[12] Chorin, A. J., A numerical method for solving incompressible viscous flow problems, Journal of Computational Physics, 135, pp. 118-125, 1997.

[13] Veijola, T., Kuisma, H., Lahdenpera, J. \& Ryhanen, T., Equivalent circuit model of the squeezed gas film in a silicon accelerometer, Sensors and Actuators, A 48, pp. 239-248, 1995.

[14] Van Leer, B., Towards the ultimate conservative difference scheme. V. A second-order sequel to Godunov's method, Journal of Computational Physics, 32(1), pp. 101-136, 1979.

[15] Briley, W. R., Neerarambam, S. S. \& Whitfield, D. L., Implicit lowerupper/approximate-factorization schemes for incompressible flows, Journal of Computational Physics, 128, pp. 32-42, 1996.

[16] Yuan, L., Comparison of implicit multigrid schemes for three-dimensional incompressible flows, Journal of Computational Physics, 177, pp. 134-155, 2002.

[17] Thomas, P. D. \& Lombard, C. K., Geometric conservation law and its application to flow computations on moving grids, AIAA Journal, 17, pp. 1030-1037, 1979.

[18] Wall, W. A., Fluid-struktur-interaktion mitstabilisieten finiten elementen. phD thesis, Universität Stuttgart, institute für Baustatik, 2000. 